\title{
Upper Jugular Lymph Node Group (Sublevels IIA and IIB)
}

National Cancer Institute

\section{Source}

National Cancer Institute. Upper Jugular Lymph Node Group (Sublevels IIA and IIB). NCI

Thesaurus. Code C132510.

Lymph nodes located around the upper third of the internal jugular vein and adjacent spinal accessory nerve, extending from the level of the skull base (above) to the level of the inferior border of the hyoid bone (below). The anterior (medial) boundary is the stylohyoid muscle (the radiologic correlate is the vertical plane defined by the posterior surface of the submandibular gland) and the posterior (lateral) boundary is the posterior border of the sternocleidomastoid muscle. Sublevel IIA nodes are located anterior (medial) to the vertical plain defined by the spinal accessory nerve. Sublevel IIB nodes are located posterior (lateral) to the vertical plane defined by the spinal accessory nerve (the radiologic correlate is the lateral border of the internal jugular on a contrast-enhanced CT scan). The upper jugular nodes are at greatest risk for harboring metastases from cancers arising from the oral cavity, nasal cavity, nasopharynx, oropharynx, hypopharynx, larynx, and parotid gland. (AJCC 8th ed.) 